\title{
Precipitous delivery after induction of combined spinal epidural analgesia
}

\author{
Krzysztof M. Kuczkowski · Claudia L. Fernández
}

Received: 28 March 2010/ Accepted: 6 May 2010/Published online: 19 May 2010

(C) Springer-Verlag 2010

The combined spinal epidural (CSE) analgesia for labor pain has attained widespread popularity in obstetric anesthesia worldwide [1]. While there still remains some concern about intentional dural puncture with the spinal needle, the CSE technique offers a number of advantages to the laboring woman $[1,2]$. First, the CSE technique provides the possibility of combining rapid onset of subarachnoid analgesia with the flexibility of continuous epidural analgesia. Second, the reliability of the CSE technique improves the quality of analgesia and overall maternal satisfaction [1].

In 1999 Tsen et al. [3] first reported that cervical dilation is more rapid in nulliparous women receiving CSE compared with epidural analgesia for labor. In 2003 Kuczkowski reported similar findings observed in multiparous women who received CSE analgesia [4].

We herein report a case of 23-year-old G2P1, healthy female at term who was in spontaneous labor with cervical dilation of $3 \mathrm{~cm}$ and consented to CSE, which was performed in a standard manner. Within 7 min after induction of CSE, complete cervical dilation was accomplished and uneventful vaginal delivery of a healthy neonate followed. Both maternal and fetal/neonatal weight were normal.

The mechanism by which CSE may be associated with increased rate of cervical dilation relative to epidural analgesia for labor is not known. Although it is difficult to

K. M. Kuczkowski (ه)

Department of Obstetrics and Gynecology,

University Medical Center of El Paso,

4800 Alberta Avenue, El Paso, Texas, USA

e-mail: kmkuczkowski@gmail.com

\section{L. Fernández}

Department of Anesthesiology, Juan A. Fernández Hospital,

Buenos Aires, Argentina know if rapid cervical dilation in our patient following induction of CSE is a physiologic effect of the technique or purely a result of patient selection, we believe that rapid onset of pain relief with CSE might have decreased the maternal catecholamine levels, which subsequently increased uterine activity leading to rapid cervical dilatation and precipitous delivery.

Additionally other authors have noted that the spinal (subarachnoid) component of the CSE might allow, at least initially, for a reduction in the total dose of local anesthetic required to control labor pain (when compared with conventional epidural analgesia), and subsequently lesser degree of a motor block.

Further studies are needed to confirm these findings.

Conflict of interest statement None.

\section{References}

1. Kuczkowski KM (2004) Ambulation with combined spinalepidural labor analgesia: the technique. Acta Anaesthesiol Belg 55:29-34

2. Okutomi T, Saito M, Mochizuki J, Kuczkowski KM (2009) Combined spinal-epidural analgesia for labor pain: best timing of epidural infusion following spinal dose. Arch Gynecol Obstet 279:329-334

3. Tsen LC, Thue B, Datta S, Segal S (1999) Is combined spinal epidural analgesia associated with more rapid cervical dilation in nulliparous patients when compared with conventional epidural analgesia? Anesthesiology 91:920-925

4. Kuczkowski KM (2003) Combined spinal-epidural analgesia and cervical dilation: is there an association? Acta Anaesthesiol Scand 47:1305 